Revista Brasil. Bot., V.31, n.2, p.253-261, abr.-jun. 2008

\title{
Germinação de sementes de Drymaria cordata (L.) Willd. ex Roem \& Schult.: efeito do potencial hídrico
}

\author{
VICTOR JOSÉ MENDES CARDOSO ${ }^{1,2}$ e FERNANDO JORGE MUNIZ PEREIRA ${ }^{1}$
}

(recebido: 10 de agosto de 2006; aceito: 08 de maio de 2008)

\begin{abstract}
Germination of Drymaria cordata (L.) Willd. ex Roem \& Schult. seeds: effect of water potential). Mathematical models based on thermal time (degree day) and hydrotime $\left(\psi_{\mathrm{w}}\right.$ day) can be used as an input to more general models of seed germination and seedling emergence in the field, and may be an important tool for understanding weed biology and control. Germination of Drymaria cordata seeds in response to reduced water potentials in the medium was evaluated by using the hydrotime model. Both the germinability and germination rate decreased linearly with the reduction of the water potential $\left(\psi_{\mathrm{w}}\right)$, attaining values near a zero at $-0.8 \mathrm{MPa}$. The rate of decrease was higher at optimum temperature as compared to suband supra-optimum temperatures. The mean base water potential $\left(\psi_{\mathrm{wb}}\right)$ was similar between sub- $\left(19{ }^{\circ} \mathrm{C}\right)$ and supra-optimum $\left(32{ }^{\circ} \mathrm{C}\right)$ temperatures, and was higher (less negative) at optimum temperature $\left(25^{\circ} \mathrm{C}\right)$, showing that $D$. cordata seeds are less sensitive to reduced water potential at $19{ }^{\circ} \mathrm{C}$ than at $25^{\circ} \mathrm{C}$. Hydrotime was higher for seeds germinated at sub-optimum than at optimum temperature, showing that the germination rate for a given water potential is higher at optimum temperature. The hydrotime required for seeds to complete germination was higher at supra-optimum than at optimum temperature, and lower at supra-optimum than at sub-optimum temperature. In general, hydrotime was relatively constant among subpopulations. The hydrotime model can well describe the effects of water potential on the time-course of seed germination of D. cordata, particularly at sub- and supra-optimum temperatures.
\end{abstract}

Key words - hydrotime, modelling, temperature, weeds

RESUMO - (Germinação de sementes de Drymaria cordata (L.) Willd. ex Roem \& Schult.: efeito do potencial hídrico). Modelos matemáticos baseados no conceito de graus dia ("thermal-time") e $\psi_{\mathrm{w}}$ dia ("hydrotime") podem ser usados para a elaboração de modelos mais gerais sobre a germinação e emergência de plântulas no campo, podendo ser uma importante ferramenta para estudos sobre a biologia de plantas daninhas e seu controle. Neste trabalho, avaliou-se a germinação de sementes de $D$. cordata em resposta ao potencial hídrico $\left(\psi_{\mathrm{w}}\right)$, usando-se o modelo $\psi_{\mathrm{w}}$ dia. Tanto a germinabilidade como a velocidade de germinação decresceram linearmente com a redução do $\psi_{\mathrm{w}}$, atingindo valores próximos a zero em - $0.8 \mathrm{MPa}$. Em temperatura ótima, a taxa de queda na germinação foi maior em comparação com as temperaturas sub- e supra-ótima. $\mathrm{O} \psi_{\mathrm{w}}$ base $\left(\psi_{\mathrm{wb}}\right)$ mediano foi similar entre as temperaturas sub-ótima $\left(19^{\circ} \mathrm{C}\right)$ e supra-ótima $\left(32{ }^{\circ} \mathrm{C}\right)$, mas foi maior $(\mathrm{menos}$ negativo) à temperatura ótima $\left(25^{\circ} \mathrm{C}\right)$, mostrando que sementes de $D$. cordata são menos sensíveis à redução do potencial hídrico à $19{ }^{\circ} \mathrm{C}$ do que à $25^{\circ} \mathrm{C}$. O $\psi_{\text {w }}$ dia foi maior para sementes germinadas à temperatura sub-ótima do que à temperatura ótima, mostrando que a velocidade de germinação num dado potencial hídrico é maior em temperatura ótima. A quantidade de $\psi_{\mathrm{w}}$ dia necessária para a germinação foi maior em temperatura supra-ótima do que em temperatura ótima, e menor em temperatura supra-ótima do que em sub-ótima. Em geral, $\psi_{\mathrm{w}}$ dia foi relativamente constante entre as diferentes sub-populações. O modelo de $\psi_{\mathrm{w}}$ dia pode descrever bem o efeito do potencial hídrico sobre as curvas de germinação (porcentagem acumulada $\mathrm{x}$ tempo), especialmente às temperaturas sub-ótima e supra-ótima.

Palavras-chave - modelagem, plantas invasoras, temperatura, $\psi_{\mathrm{w}}$ dia

\section{Introdução}

Do ponto de vista fisiológico, o processo de germinação inicia-se com a captação de água pela semente e termina com o crescimento do embrião, que se torna visível quando uma parte deste transpassa o envoltório da semente (Bewley 1997). Considerando-se a germinação como a

\footnotetext{
1. Universidade Estadual Paulista, Instituto de Biociências, Departamento de Botânica, Caixa Postal 178, 13506-900 Rio Claro, SP, Brasil

2. Autor para correspondência: victorjc@ @rc.unesp.br
}

protrusão de uma parte do embrião através dos tegumentos, e considerando-se que essa é uma resposta do tipo tudoou-nada, a germinação tem sido descrita por intermédio de curvas que mostram a germinação acumulada em diferentes intervalos de tempo. $\mathrm{O}$ formato dessas curvas indica, em última análise, a distribuição dos tempos de germinação de uma população de sementes.

Os dois principais fatores ambientais determinantes da germinação são o potencial hídrico $\left(\psi_{w}\right)$ e a temperatura. A disponibilidade de água, representada pelo $\psi_{\mathrm{w}}$ é um fator limitante da capacidade e da velocidade de germinação, já que o crescimento do embrião durante o 
processo germinativo não ocorre se não houver pressão de turgescência intracelular suficiente para a sua expansão (Welbaum et al. 1998). Em relação à temperatura, a germinação é balizada pelas temperaturas mínima ou base $(\mathrm{Tb})$, ótima (To) e máxima $\left(\mathrm{T}_{\mathrm{M}}\right)$. Tb e $\mathrm{T}_{\mathrm{M}}$ representam, respectivamente, os limites inferior $\mathrm{e}$ superior, além dos quais não ocorre germinação; enquanto que To é a temperatura (ou intervalo térmico) na qual a germinação é maior e mais rápida (Labouriau 1983, Bradford 2002). A faixa térmica limitada por Tb e To é denominada infra-ótima ou sub-ótima, ao passo que o intervalo entre To e $\mathrm{T}_{\mathrm{M}}$ é chamado supra-ótimo. Em muitos casos, observa-se que nesses intervalos ocorre uma relação linear, ou aproximadamente linear, entre velocidade de germinação e temperatura (Labouriau 1983).

Diversos trabalhos têm mostrado também que sementes embebidas em água, e mantidas em diferentes temperaturas constantes, germinam em função de um determinado número de "graus dia" acumulados $\left(\theta_{g}\right)$, de acordo com a equação:

$$
\left.\theta_{g}=\left(T-T_{b}\right) t_{g}, \quad \text { (equação } 1\right)
$$

onde $\mathrm{T}_{\mathrm{b}}$ é a temperatura mínima ou base para a germinação de uma dada fração percentual $\mathrm{g}, \mathrm{t}_{\mathrm{g}}$ é o intervalo de tempo para a germinação da fração $\mathrm{g}$ e $\mathrm{T}$ é a temperatura experimental sub-ótima (Dahal \& Bradford 1994).

O modelo descrito por Gummerson (1986) permite tratar o efeito do $\psi_{\mathrm{w}}$ sobre o decurso da germinação de modo similar àquele da temperatura, levando-se em conta que tanto a redução do $\psi_{\mathrm{w}}$ do meio, como a redução da temperatura, afetam as curvas de germinação de maneira similar. Gummerson (1986) propôs que as respostas germinativas ao $\psi_{\mathrm{w}}$ podem ser descritas em escala de " $\psi_{\mathrm{w}}$ dia" (hydrotime) análoga à escala de graus dia, de acordo com o modelo:

$$
\theta_{H}=\left(\psi-\psi_{w b}(g)\right) t_{g}, \quad(\text { equação } 2)
$$

onde: $\theta_{\mathrm{H}}$ é uma constante que indica a quantidade de " $\psi_{\mathrm{w}}$ dia" acumulada, necessária para a germinação; $\psi_{\mathrm{wb}}(\mathrm{g})$ é o potencial hídrico limite ou base, a partir do qual a germinação de uma fração porcentual g deixa de ocorrer e; $\mathrm{t}_{\mathrm{g}}$ é o tempo real necessário para a germinação da fração $g$.

Assim como no conceito de graus dia, o princípio do modelo " $\psi_{\mathrm{w}}$ dia" baseia-se na existência de "subpopulações" ou frações que respondem de maneira diferente à flutuação do potencial hídrico do meio, ou seja, $\psi_{\mathrm{wb}}$ varia entre as populações (Bradford 1995).

Modelos baseados no conceito de graus dia e $\psi_{\mathrm{w}}$ dia podem ser usados para se prever a emergência de plântulas no campo (Finch-Savage \& Phelps 1993, Battla \& Benech-Arnold 2006), o que, no caso de plantas daninhas ou invasoras, pode ser uma informação importante visando seu manejo e controle. Drymaria cordata (Cariophyllaceae) é uma planta anual, herbácea, que produz grande quantidade de sementes, as quais podem constituir um banco de sementes no solo. É uma invasora comum em várzeas e locais mais ou menos sombreados, infestando jardins, pastagens, hortas e terrenos baldios (Lorenzi 1982). Em laboratório, suas sementes germinam entre 17 e $34{ }^{\circ} \mathrm{C}$, apresentando temperatura ótima ao redor de $25^{\circ} \mathrm{C}$ (Pereira \& Cardoso, dados não publicados). Neste trabalho, avaliou-se a germinação de sementes de D. cordata em resposta ao potencial hídrico, em três diferentes temperaturas (subótima, ótima e supra-ótima), aplicando-se o modelo de $\psi_{\mathrm{w}}$ dia (hydrotime), e verificando-se a eficiência desse modelo em descrever a germinação da semente em relação a variações no potencial hídrico do meio.

\section{Material e métodos}

Foram utilizadas sementes de Drymaria cordata (Caryophyllaceae) coletadas em Rio Claro, SP, a partir de plantas que ocorriam espontaneamente em gramados. A coleta foi feita manualmente, sendo os frutos colocados para secar ao ar livre em bandejas, para a liberação das sementes, as quais foram separadas por peneiramento e armazenadas em frascos de plástico protegidos da luz, à temperatura de $25^{\circ} \mathrm{C}$.

Os ensaios foram realizados em câmara de germinação, colocando-se as sementes em placas de Petri de $60 \mathrm{~mm}$ de diâmetro, sobre dois discos de papel de filtro saturados com água destilada ou solução de polietilenoglicol (PEG 6000). Para minimizar a evaporação, as placas foram distribuídas ao acaso dentro de bandejas de plástico revestidas internamente com papel de filtro umedecido com água destilada, e cobertas com uma chapa de vidro liso transparente. As placas foram mantidas sob luz fluorescente branca contínua, com irradiância média de $34,6 \mu \mathrm{mol} \mathrm{m} \mathrm{m}^{-2} \mathrm{~s}^{-1}$, medida com sensor de radiação acoplado a um DataLogger LI-1000 (LI-COR). Os potenciais hídricos teóricos (-0,2 a -0,8 MPa) foram obtidos utilizando-se soluções com diferentes concentrações de PEG 6000 preparadas de acordo com Michel \& Kaufmann (1973). Quando necessário, as soluções nas placas eram trocadas para se minimizar o efeito da evaporação. Para cada tratamento foram utilizadas três repetições de 100 sementes cada. Os experimentos foram conduzidos em três temperaturas constantes: $19{ }^{\circ} \mathrm{C}$ (temperatura sub-ótima), $25^{\circ} \mathrm{C}$ (ótima) e $32{ }^{\circ} \mathrm{C}$ (supra-ótima). As temperaturas registradas na câmara apresentaram uma variação de $\pm 0,5^{\circ} \mathrm{C}$ em relação à temperatura estabelecida.

As contagens foram feitas diariamente, utilizando-se a protrusão radicular como critério de germinação. As 
respostas de germinação aos diferentes potenciais hídricos $\left(\psi_{\mathrm{w}}\right)$ foram analisadas de acordo com o modelo representado na equação 2 (Gummerson 1986, Bradford 2002). Os parâmetros do modelo foram estimados de acordo com a equação (Bradford 1995):

$$
\operatorname{probit}(g)=\left[\psi_{w}-\left(\theta_{H} / t_{g}\right)-\psi_{w b} M\right] / \sigma \quad(\text { equação } 3)
$$

onde: probit(g) é a transformação probítica das porcentagens acumuladas de germinação $g ; \psi_{\mathrm{w}}$ é o potencial hídrico do meio; $\theta_{\mathrm{H}}$ é a constante " $\psi_{\mathrm{w}}$ dia"; $\mathrm{t}_{\mathrm{g}}$ é o tempo necessário para a germinação de $\mathrm{g} ; \psi_{\mathrm{wb}} \mathrm{M}$ é o potencial hídrico base mediano, ou seja, é o $\psi_{\mathrm{w}}$ no qual a fração é igual a $50 \%$ e; $\sigma$ é o desvio padrão da distribuição de $\psi_{\text {wb }}$ dentro da população.

Assim, para cada temperatura, os parâmetros $\theta_{\mathrm{H}}, \psi_{\mathrm{wb}} \mathrm{M}$ e $\sigma$ foram obtidos a partir da regressão linear do conjunto das porcentagens de germinação acumuladas obtidas com os diferentes potenciais hídricos, transformadas em probit, em $\psi-\left(\theta_{\mathrm{H}} / \mathrm{t}_{\mathrm{g}}\right)$. Para a estimativa de $\mathrm{t}_{\mathrm{g}}$, as curvas de germinação originais foram ajustadas de acordo com o modelo de Weibull (Dumur et al. 1990). Os parâmetros foram então usados na equação (3) para a obtenção das curvas de germinação previstas pelo modelo para os diferentes potenciais hídricos testados (Bradford 1995).

A normalização das curvas de germinação com base na quantidade acumulada de $\psi_{\mathrm{w}}$ dia foi feita multiplicando-se os tempos reais de germinação pelo fator $1-\psi / \psi_{\mathrm{wb}}(\mathrm{g})$, onde o termo $\psi_{\mathrm{wb}}(\mathrm{g})$ é o potencial base correspondente a cada porcentagem g, obtido também a partir da regressão linear de probit $(\mathrm{g})$ sobre $\psi-\left(\theta_{\mathrm{H}} / \mathrm{t}_{\mathrm{g}}\right)$ (Bradford 1995).

\section{Resultados}

Inicialmente são apresentadas as curvas de porcentagem acumulada de germinação das sementes nos seguintes potenciais hídricos teóricos $\left(\psi_{\mathrm{w}}\right): 0,0 \mathrm{MPa}$ (controle); -0,2 MPa; -0,4 MPa; -0,6 MPa e -0,8 MPa (figura 1). A germinação foi nula em solução a -1,0 MPa, nas três temperaturas. Observa-se que a germinabilidade (G), de um modo geral, decresceu proporcionalmente com a redução do $\psi_{\mathrm{w}}$; entretanto, deve-se destacar que a $25{ }^{\circ} \mathrm{C}$ a curva mostrou-se ascendente nos tratamentos de -0,2 MPa a -0,6 MPa, mesmo após 30 dias de

Figura 1. Porcentagens acumuladas de germinação (G \%) de sementes de Drymaria cordata em diferentes potenciais hídricos $\left(\psi_{\mathrm{w}}\right)(\diamond=$ água destilada, $0,0 \mathrm{MPa}, \square=-0,2 \mathrm{MPa}$, $\Delta=-0,4 \mathrm{MPa}, \mathrm{O}=-0,6 \mathrm{MPa}, *=-0,8 \mathrm{MPa})$, a $19^{\circ} \mathrm{C}(\mathrm{A})$, $25{ }^{\circ} \mathrm{C}(\mathrm{B})$ e $32{ }^{\circ} \mathrm{C}(\mathrm{C})$, com as respectivas curvas ajustadas pela função de Weibull (linhas contínuas): $\psi=A\{1-\exp$ $\left.\left[-k(x-z)^{d}\right]\right\}$. D: germinabilidade (\% final de germinação) em relação ao potencial hídrico, a $19^{\circ} \mathrm{C}(\boldsymbol{\square}), 25^{\circ} \mathrm{C}(\square)$ e $32{ }^{\circ} \mathrm{C}(\triangle)$. Linhas verticais no $30^{\circ} \mathrm{dia}$, representam intervalos de confiança $(P=0,95)$.
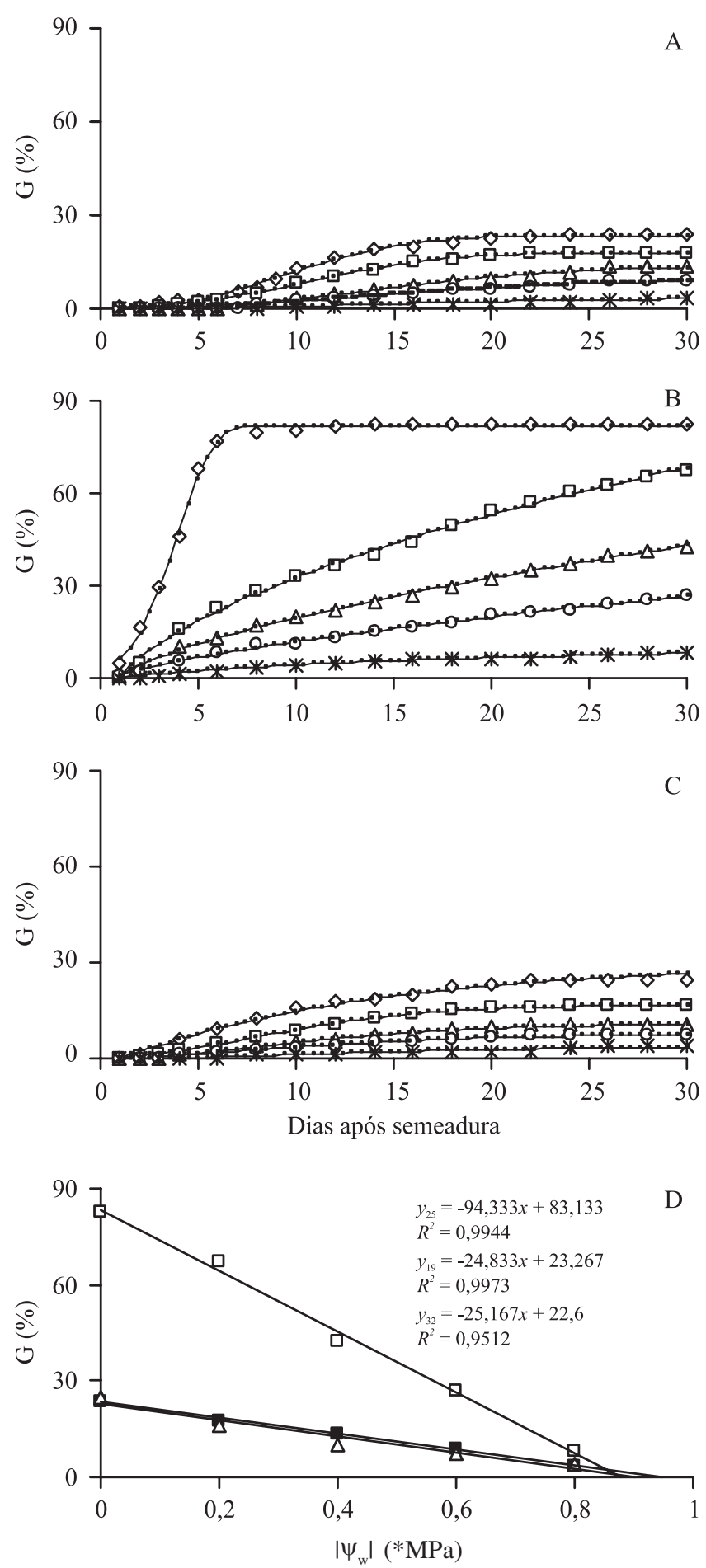

Figure 1. Cumulative germination percentage (G\%) of Drymaria cordata seeds in different water potentials $\left(\psi_{\mathrm{w}}\right)$ $(\diamond=$ distilled water, $0,0 \mathrm{MPa}, \square=-0,2 \mathrm{MPa}, \Delta=-0,4 \mathrm{MPa}$, $\mathrm{O}=-0,6 \mathrm{MPa}, *=-0,8 \mathrm{MPa})$, at $19{ }^{\circ} \mathrm{C}(\mathrm{A}), 25^{\circ} \mathrm{C}(\mathrm{B})$ and $32{ }^{\circ} \mathrm{C}(\mathrm{C})$, with the curves predicted by the Weibull function (solid lines): $\psi=A\left\{1-\exp \left[-k(x-z)^{d}\right]\right\}$. D: germinability (final \%) related to osmotic potential, at $19{ }^{\circ} \mathrm{C}(\boldsymbol{\square}), 25^{\circ} \mathrm{C}$ $(\square)$ and $32{ }^{\circ} \mathrm{C}(\Delta)$. Vertical lines on $30^{\circ}$ day denote 0,95 confidence intervals $(P=0,95)$. 
incubação (figura 1B). A redução de $\mathrm{G}$ em função do $\psi_{\mathrm{w}}$ apresentou tendência claramente linear, conforme pode ser visto na figura $1 \mathrm{D}$, manifestando-se numa taxa bem mais elevada à temperatura de $25^{\circ} \mathrm{C}$, em comparação com as temperaturas sub e supra-ótima (notar os respectivos coeficientes de inclinação na figura 1D).

As curvas apresentadas nas figuras $1 \mathrm{~A}, 1 \mathrm{~B}$ e $1 \mathrm{C}$ descrevem a distribuição das porcentagens acumuladas de germinação ao longo do tempo, de acordo com o modelo de Weibull, $\psi=A\left\{1-\exp \left[-k(x-z)^{d}\right]\right\}$, onde $\mathrm{A}, \mathrm{k}, \mathrm{z}$ e d são constantes, $\psi$ é a porcentagem e x o tempo. A partir das curvas, determinou-se a recíproca dos tempos necessários para a germinação de uma dada fração percentual $\psi$, tomando-se por base a expressão $x=\left\{\left[\left(\ln (A /(A-\psi))^{1 / d}\right] / k\right\}+z\right.$. Essas recíprocas $(1 / \mathrm{x})$, representando as velocidades de germinação, foram plotadas como uma função do $\psi_{\mathrm{w}}$ (figura 2). Considerando-se as germinabilidades relativamente baixas observadas nos menores $\psi_{\mathrm{w}}$, particularmente nas temperaturas de 19 e $32{ }^{\circ} \mathrm{C}$, desconsiderou-se o tratamento de $-0,8 \mathrm{MPa}$ e atribuiu-se a $\psi$ valores de $5 \%$ a $50 \%$, com intervalos de $5 \%$. Observou-se para as três temperaturas que, assim como para a variável germinabilidade, a velocidade de germinação decresceu com a redução do $\psi_{\mathrm{w}}$ do meio, sendo que essa redução também pôde ser descrita por retas, uma para cada fração percentual. De modo geral, com exceção da fração 5\% $(\psi=5)$, as curvas puderam ser representadas por retas paralelas (figuras 2A, 2B e 2C). A recíproca da inclinação de cada uma dessas retas representa a constante $\psi_{\mathrm{w}}$ dia (hydrotime $=\theta_{\mathrm{H}}$ ), ao passo que o intercepto com o eixo das abscissas indica o $\psi_{\text {wb }}$ para a fração percentual considerada. A relação entre cada $\psi_{\text {wb }}$ estimado desse modo e a respectiva fração percentual é apresentada na figura $3 \mathrm{~A}$, onde se observa um decréscimo de $\psi_{\mathrm{wb}}$ com o aumento da fração percentual. A taxa de decréscimo não foi uniforme, dependendo da temperatura. Por exemplo, a $25^{\circ} \mathrm{C}$ não houve diferença significativa entre os valores de $\psi_{\text {wb }}$ estimados para as frações de $5 \%$ a $25 \%$ (figura $3 \mathrm{~A}$ ). Os valores médios de $\psi_{\mathrm{wb}}$ variaram de -1,0 MPa a -0,6 MPa, de -0,7 MPa a -0,4 MPa, e de $-0,9 \mathrm{MPa}$ a $-0,4 \mathrm{MPa}$, respectivamente para as temperaturas de 19,25 e $32{ }^{\circ} \mathrm{C}$. Quanto aos valores de $\psi_{\mathrm{w}}$ dia, com exceção da fração $5 \%$, praticamente não houve diferença significativa entre as várias frações. Os valores médios de $\psi_{\mathrm{w}}$ dia, desconsiderando-se a fração $5 \%$, variaram de 5,2 a 6,7 , de 1,0 a 1,6 e de 2,5 a 3,0 , respectivamente para as temperaturas de 19,25 e $32^{\circ} \mathrm{C}$ (figura 3B).

Para se obter os valores de $\theta_{\mathrm{H}}, \psi_{\mathrm{wb}}$ mediano $\left(\psi_{\mathrm{wb}} \mathrm{M}\right)$ e o desvio padrão de $\psi_{\mathrm{wb}}(\sigma)$, as porcentagens
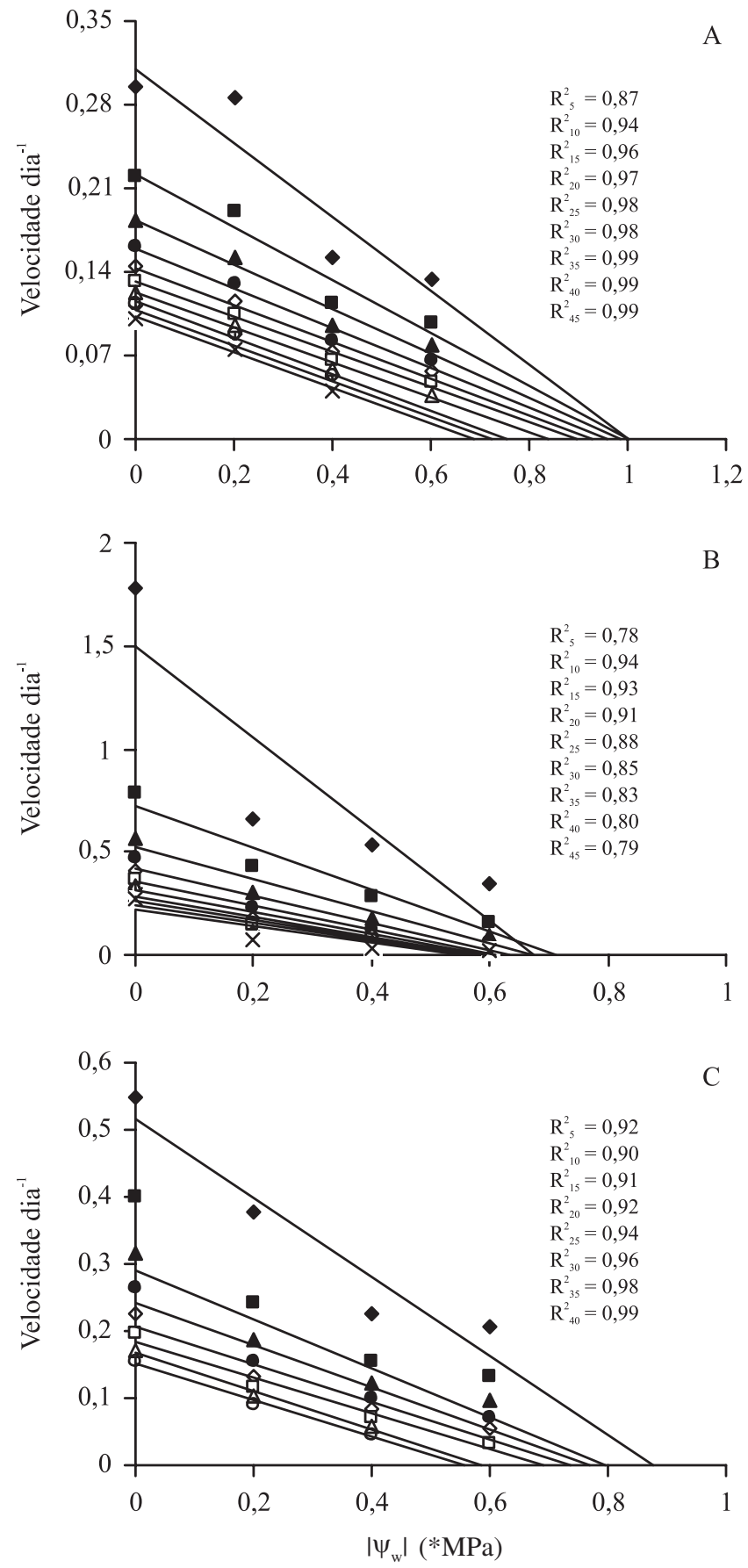

Figura 2. Relação entre velocidade de germinação de diferentes sub-populações $(5 \%=\diamond ; 10 \%=\mathbf{\square} ; 15 \%=\boldsymbol{\Delta}$; $20 \%=\bullet ; 25 \%=\diamond ; 30 \%=\square ; 35 \%=\Delta ; 40 \%=0 ; 45 \%=* ;$ $50 \%=x)$ de sementes de Drymaria cordata e potencial hídrico $\left(\psi_{\mathrm{w}}\right)$, a $19{ }^{\circ} \mathrm{C}(\mathrm{A}), 25^{\circ} \mathrm{C}(\mathrm{B})$ e $32{ }^{\circ} \mathrm{C}(\mathrm{C})$.

Figure 2. Relation between germination rates for specific percentages $(5 \%=\boldsymbol{\nabla} ; 10 \%=\mathbf{\square} ; 15 \%=\mathbf{\Delta} ; 20 \%=\bullet ; 25 \%=$ $\diamond ; 30 \%=\square ; 35 \%=\Delta ; 40 \%=0 ; 45 \%=* ; 50 \%=x$ ) of Drymaria cordata L. seeds and water potential $\left(\psi_{\mathrm{w}}\right)$, at $19{ }^{\circ} \mathrm{C}$ (A), $25{ }^{\circ} \mathrm{C}(\mathrm{B})$ and $32{ }^{\circ} \mathrm{C}(\mathrm{C})$. 

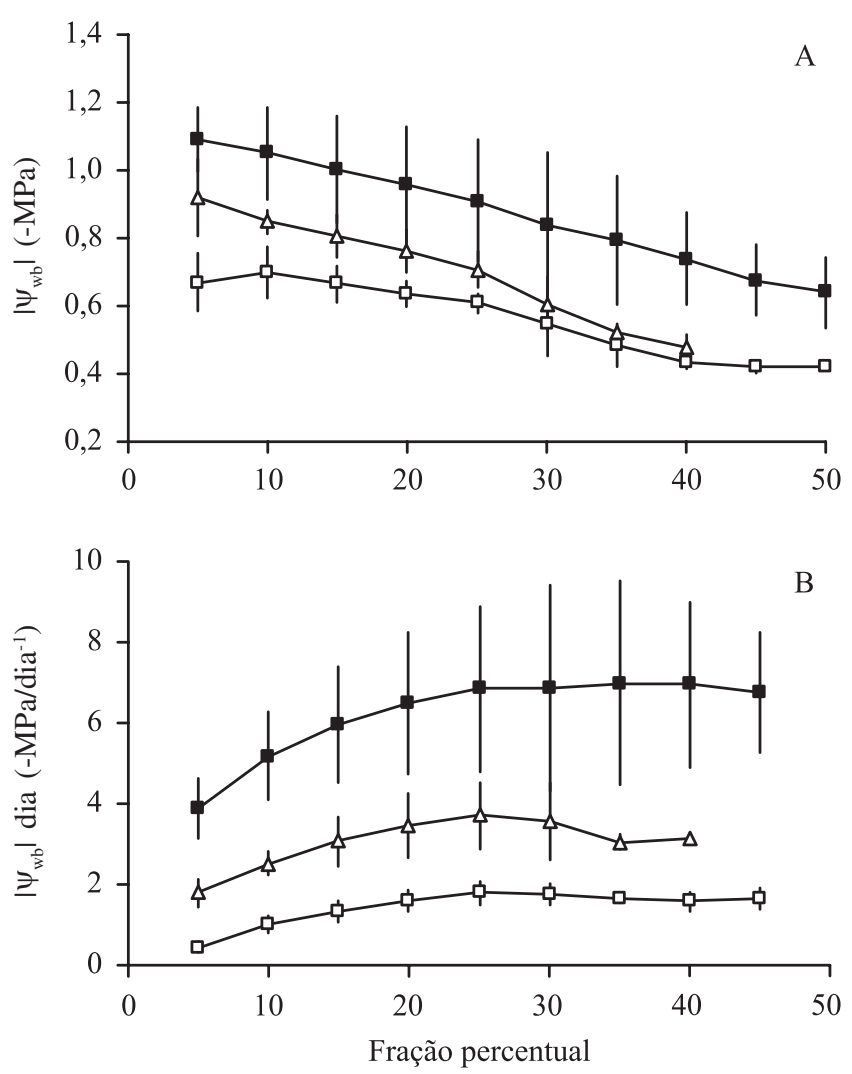

Figura 3. Variação do $\psi_{\mathrm{wb}}(\mathrm{A})$ e $\psi_{\mathrm{w}}$ dia (B) em relação a diferentes frações percentuais de sementes de Drymaria cordata germinadas a $19{ }^{\circ} \mathrm{C}(\boldsymbol{\square}), 25^{\circ} \mathrm{C}(\square)$ e $32{ }^{\circ} \mathrm{C}(\Delta)$. Barras verticais $=$ intervalos de confiança $(P=0,05)$.

Figure 3. Variation of $\psi_{\mathrm{wb}}(\mathrm{A})$ and hydrotime (B) related to different sub-populations of Drymaria cordata seeds germinated at $19^{\circ} \mathrm{C}(\boldsymbol{\square}), 25^{\circ} \mathrm{C}(\square)$ and $32^{\circ} \mathrm{C}(\Delta)$. Vertical bars $=$ confidence intervals $(P=0.05)$.

acumuladas de germinação, obtidas nas diversas contagens efetuadas ao longo do tempo, em todos os tratamentos, foram transformadas em probits e plotadas contra os respectivos valores de $\psi-\theta_{\mathrm{H}} / \mathrm{t}_{\mathrm{g}}$ (figura 4 ), termo que segundo a equação 2 é equivalente a $\psi_{\mathrm{wb}}$. A partir da estimativa inicial de $\theta_{\mathrm{H}}$, para cada temperatura, diferentes valores de $\theta_{\mathrm{H}}$ foram experimentados até a obtenção dos coeficientes de regressão mais elevados, os quais estão expressos nas figuras 4A, 4B e 4C. Porcentagens de germinação inferiores a $5 \%$ não foram consideradas na distribuição dos pontos apresentados na figura 4. Usando-se esse procedimento, os seguintes valores dos parâmetros $\theta_{\mathrm{H}}, \psi_{\mathrm{wb}} \mathrm{M}$ e $\sigma$ foram obtidos: a) $19{ }^{\circ} \mathrm{C}, \theta_{\mathrm{H}}=5,9 \mathrm{MPa}$.dia, $\psi_{\mathrm{wb}}=-0,79 \mathrm{MPa}, \sigma=0,73$ $\mathrm{MPa}$; b) $25^{\circ} \mathrm{C}, \theta_{\mathrm{H}}=1,2 \mathrm{Mpa}$ dia, $\psi_{\text {wb }} \mathrm{M}=-0,46 \mathrm{MPa}$, $\sigma=0,33 \mathrm{MPa}$; c) $32{ }^{\circ} \mathrm{C}, \theta_{\mathrm{H}}=3,0 \mathrm{Mpa}$ dia, $\psi_{\mathrm{wb}} \mathrm{M}=$ $-0,82 \mathrm{MPa}, \sigma=0,7 \mathrm{MPa}$.
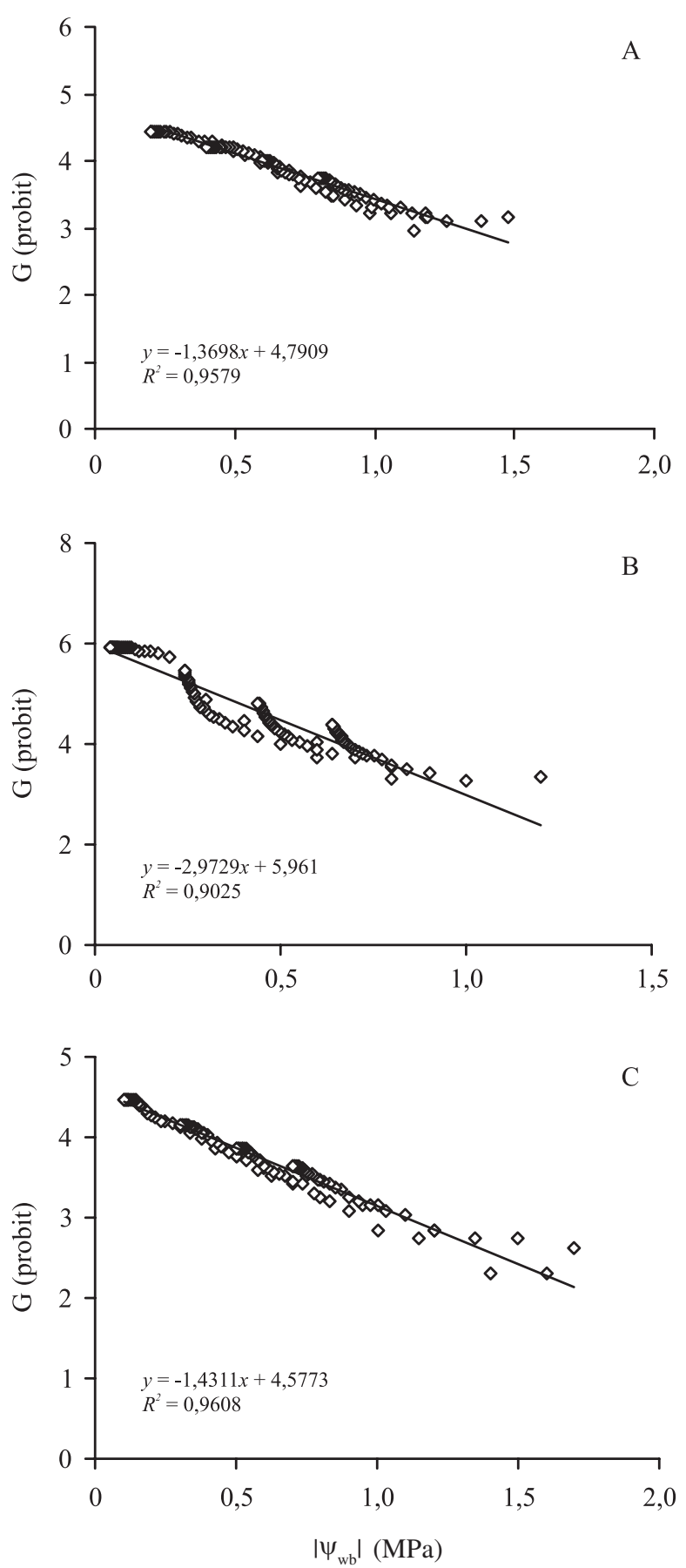

Figura 4. Regressão das porcentagens diárias acumuladas (G), transformadas em probits, de sementes de Drymaria cordata germinadas nos potenciais hídricos de 0,0 $\mathrm{MPa}$, -0,2 MPa, -0,4 MPa, -0,6 MPa e -0,8 MPa, sobre os respectivos $\psi_{\mathrm{wb}}\left(=\psi_{w}-\left(\theta_{H} / t_{g}\right)\right) . \mathrm{A}=19{ }^{\circ} \mathrm{C}, \mathrm{B}=25^{\circ} \mathrm{C}$ e $\mathrm{C}=32{ }^{\circ} \mathrm{C}$.

Figure 4. Regression on $\psi_{\mathrm{wb}}\left(=\psi_{\mathrm{w}}-\left(\theta_{\mathrm{H}} / \mathrm{t}_{\mathrm{g}}\right)\right)$ of the probit transformed cumulative daily percentages $(\mathrm{G})$ of Drymaria cordata seeds in water potentials of $0,0 \mathrm{MPa},-0,2 \mathrm{MPa}$, $-0,4 \mathrm{MPa},-0,6 \mathrm{MPa}$ and $-0,8 \mathrm{MPa} . \mathrm{A}=19{ }^{\circ} \mathrm{C}, \mathrm{B}=25^{\circ} \mathrm{C} \mathrm{e}$ $\mathrm{C}=32{ }^{\circ} \mathrm{C}$. 
Esses parâmetros foram usados no modelo representado pela equação 3 , a partir do qual foram elaboradas as curvas apresentadas nas figuras $5 \mathrm{~A}, 5 \mathrm{C}$ e 5E. Para efeito de comparação, essas curvas são apresentadas juntamente com os pontos que mostram a evolução das porcentagens de germinação observadas e corrigidas. Essa correção foi feita multiplicando-se as porcentagens reais pelo fator $(100 / 82,3)$, considerando-se que a germinabilidade máxima do lote de sementes, obtida à temperatura ótima, foi de $82,3 \%$ (figura 1B).
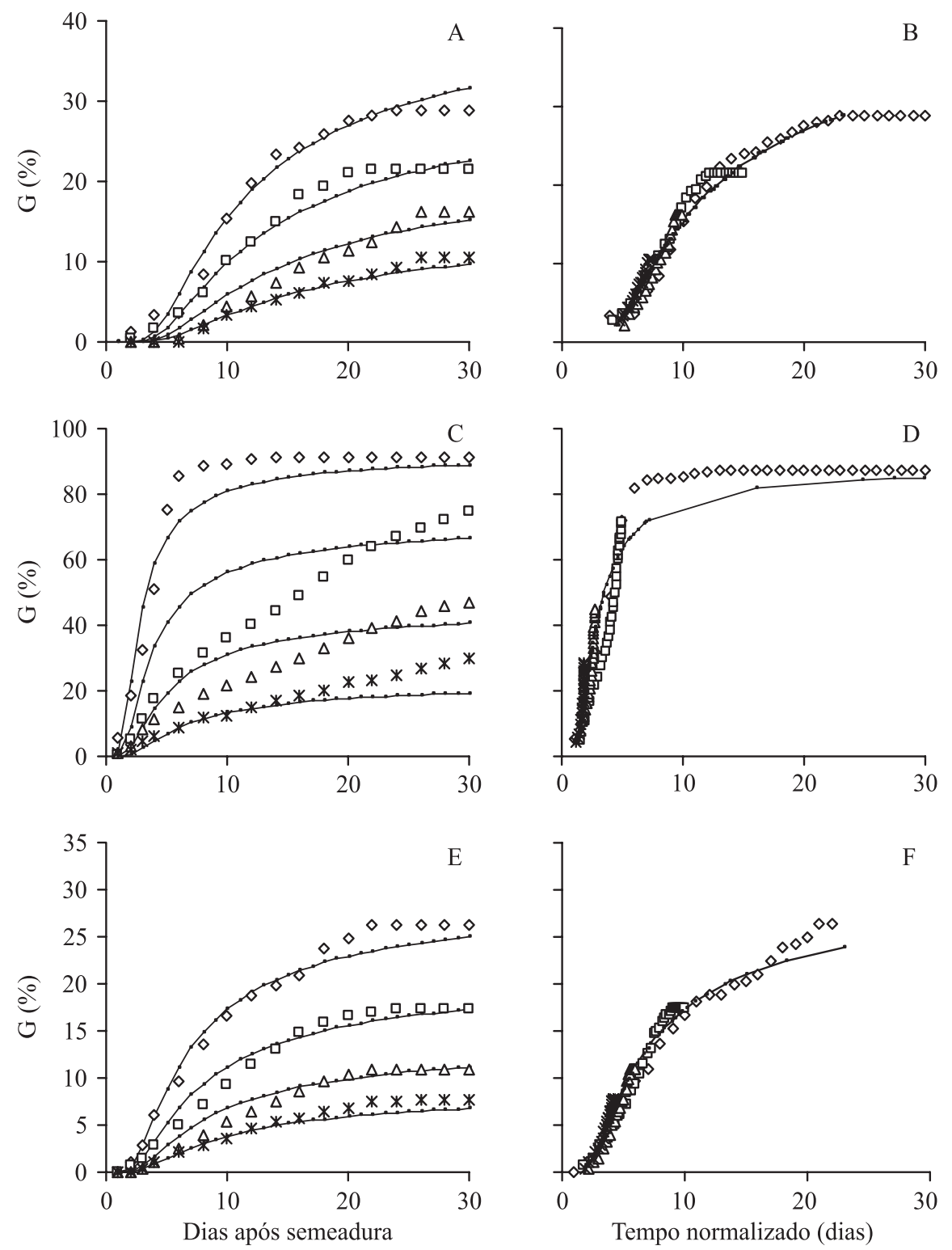

Figura 5. Porcentagens acumuladas de germinação $(\mathrm{G})$ de sementes de Drymaria cordata em diferentes potenciais hídricos $\left(\psi_{\mathrm{w}}\right)(\diamond=0 \mathrm{MPa}, \square=-0,2 \mathrm{MPa}, \Delta=-0,4 \mathrm{MPa}, *=-0,6 \mathrm{MPa})$, à $19{ }^{\circ} \mathrm{C}(\mathrm{A}, \mathrm{B}), 25^{\circ} \mathrm{C}(\mathrm{C}, \mathrm{D})$ e $32{ }^{\circ} \mathrm{C}(\mathrm{E}, \mathrm{F}) . \mathrm{A}, \mathrm{C}$ e E: comparação entre os valores observados (símbolos) e os valores previstos pelo modelo, probit $(g)=\left[\psi_{w}-\left(\theta_{H} / t_{g}\right)-\psi_{w b} M\right] / \sigma$, (linhas contínuas). B, D e F: distribuição das porcentagens observadas (símbolos) em escala de tempo normalizada (tempo real multiplicado pelo fator $\left.1-\psi_{\mathrm{w}} / \psi_{\mathrm{wb}}(\mathrm{g})\right)$; e valores esperados para $\psi_{\mathrm{w}}=0 \mathrm{MPa}$ (linha contínua).

Figure 5. Cumulative germinations percentages $(\mathrm{G})$ of Drymaria cordata seeds in different water potentials $\left(\psi_{\mathrm{w}}\right)(\diamond=0 \mathrm{MPa}$, $\square=-0,2 \mathrm{MPa}, \Delta=-0,4 \mathrm{MPa}, *=-0,6 \mathrm{MPa})$, at $19{ }^{\circ} \mathrm{C}(\mathrm{A}, \mathrm{B}), 25^{\circ} \mathrm{C}(\mathrm{C}, \mathrm{D})$ and $32{ }^{\circ} \mathrm{C}(\mathrm{E}, \mathrm{F})$. A, C and E: comparison between observed values (symbols) and that predicted by the model, probit $(g)=\left[\psi_{w}-\left(\theta_{H} / t_{g}\right)-\psi_{w b} M\right] / \sigma$, (solid lines). B, D e F: distribution of observed percentages (symbols) on a normalized time scale (actual time multiplied by $1-\psi_{\mathrm{w}} / \psi_{\mathrm{wb}}(\mathrm{g})$ ); and theoretical values for $\psi_{\mathrm{w}}=0 \mathrm{MPa}$ (solid lines). 
Com exceção do tratamento a $-0,2 \mathrm{MPa}$, a $25^{\circ} \mathrm{C}$ (figura $5 C)$, não houve diferença significativa (teste $\chi^{2}, p<0.05$ ) entre as porcentagens observadas (os pontos, nos gráficos) e as esperadas (linha contínua) (figuras 5A, $5 \mathrm{C}$ e 5E). Nas figuras 5B, 5D e 5F, são apresentadas as curvas de germinação observadas, nos diferentes tratamentos, em uma escala de tempo única, obtida pela multiplicação dos tempos reais pelo fator $\left[1-\left(\psi / \psi_{\mathrm{wb}}\right)\right]$, onde $\psi_{\mathrm{wb}}$ foi calculado para cada porcentagem observada, de acordo com as relações apresentadas na figura 4. Nota-se que os pontos correspondentes aos diferentes tratamentos tendem a se sobrepor, seguindo aproximadamente a curva esperada (linha contínua) calculada para as sementes embebidas em água destilada $\left(\psi_{\mathrm{w}}=0\right)$.

\section{Discussão}

Como relatado para diversas outras espécies (El Siddig et al. 2004, Springer 2005), a germinabilidade das sementes de Drymaria cordata foi inibida por concentrações crescentes de PEG 6000 no meio, atingindo valores próximos a zero em potencial hídrico de $-0,8 \mathrm{MPa}$, resposta essa semelhante à de sementes de Pinus sylvestris L., que deixam de germinar a partir de $\psi_{\mathrm{w}}$ de $-0,8 \mathrm{MPa}$ (Tilki 2005). Em termos proporcionais, o efeito do PEG 6000 foi independente da temperatura, considerando-se que, proporcionalmente, a resposta das sementes aos diferentes $\psi_{\mathrm{w}}$ foi praticamente idêntica às temperaturas sub-ótima $\left(19^{\circ} \mathrm{C}\right)$, ótima $\left(25^{\circ} \mathrm{C}\right)$ e supraótima $\left(32{ }^{\circ} \mathrm{C}\right)$. Por outro lado, à temperatura ótima, a diminuição da germinação em função do $\psi_{\mathrm{w}}$ foi bem mais acentuada em comparação com 19 e $32{ }^{\circ} \mathrm{C}$. Comparando-se as três temperaturas em relação à geminabilidade, um outro elemento que permite a conclusão de que a resposta das sementes de $D$. cordata à redução do $\psi_{\mathrm{w}}$ do meio independe do fator térmico é o fato de que as curvas, seja na temperatura ótima, seja nas temperaturas sub e supra-ótima, apontam praticamente para o mesmo ponto nas abscissas, o qual corresponderia ao $\psi$ limite $\left(\psi_{\mathrm{wb}}\right)$. Quanto ao efeito da temperatura em si, mesmo após 30 dias de experimento, a germinação final a 19 e $32{ }^{\circ} \mathrm{C}$ não passou de $25 \%$, contudo foi superior a $80 \%$ em sementes mantidas a $25^{\circ} \mathrm{C}$. O modelo de Weibull descreveu com bastante precisão a distribuição, ao longo do período experimental, das porcentagens acumuladas de germinação das sementes de $D$. cordata, confirmando trabalhos segundo os quais a função de Weibull representa um modelo prático e adequado para se descrever curvas de germinação (Dumur et al. 1990), permitindo estimar-se os tempos necessários para a germinação de uma dada fração percentual.

Assim como a germinabilidade, a velocidade de germinação das sementes de $D$. cordata também decresceu em função da diminuição do potencial hídrico do meio. Aplicando-se modelos lineares às respostas de diferentes frações percentuais, e considerando-se que os interceptos representam o $\psi_{\mathrm{wb}}$ (Gummerson 1986), observa-se que, quanto mais "rápidas" as sementes, mais negativo o seu $\psi_{\mathrm{wb}}$. Isso pode ser visualizado pelo fato das frações percentuais mais baixas (por exemplo, $5 \%$ e $10 \%$ ) tenderem a apresentar valores de $\psi_{\mathrm{wb}}$ mais baixos. O modelo de $\psi_{\mathrm{w}}$ dia assume que $\theta_{\mathrm{H}}$ seja constante (Bradford 2002), embora tenha sido observado que, no caso de $D$. cordata, esse parâmetro pode exibir alguma variação, principalmente em relação às menores frações percentuais. Admitindo-se que $\theta_{\mathrm{H}}$ seja relativamente constante, as diferenças nas distribuições dos tempos de germinação de $D$. cordata seriam baseadas unicamente na variação dos respectivos $\psi_{\mathrm{wb}}$, uma característica intrínseca da semente (Bradford 1995). Desse modo, dentro de uma população de sementes colocadas para germinar num meio com um dado $\psi_{\mathrm{w}}$, aquelas com $\psi_{\mathrm{wb}}$ mais negativo são capazes de germinar mais rápido do que sementes com $\psi_{\mathrm{wb}}$ menos negativo. O modelo assume também que a distribuição de $\psi_{\mathrm{wb}}$ numa população segue o padrão gaussiano (Dahal \& Bradford 1990), com média e desvio padrão característicos. Para cada temperatura, combinando-se os dados numéricos (porcentagens acumuladas de germinação transformadas em probits) dos diferentes tratamentos, e plotando-os como uma função de $\left[\psi_{\mathrm{w}}-\left(\theta_{\mathrm{H}} / \mathrm{t}_{\mathrm{g}}\right)\right]$, observa-se que as curvas tendem a cair numa única linha de regressão, a partir da qual os parâmetros da distribuição de $\psi_{\text {wb }}$ puderam ser estimados. $\mathrm{O} \psi_{\mathrm{wb}} \mathrm{M}$ praticamente não diferiu comparando-se a temperatura sub-ótima $\left(19^{\circ} \mathrm{C}\right)$ com a supra-ótima $\left(32^{\circ} \mathrm{C}\right)$, mas foi maior (menos negativo) à temperatura ótima. Além disso, os valores relativamente elevados dos desvios padrão sugerem baixa homogeneidade na distribuição das frequiências relativas desse parâmetro dentro da população de $D$. cordata. Para efeito de comparação, Dahal \& Bradford (1990), trabalhando com sementes de tomate germinadas em soluções de PEG com potenciais hídricos variando de 0,0 a $0,8 \mathrm{MPa}$, obtiveram um $\psi_{\mathrm{wb}} \mathrm{M}=-0,65 \mathrm{MPa}$ e $\sigma= \pm 0,13 \mathrm{MPa}$, enquanto Toselli \& Casanave (2005), trabalhando com algodão, observaram um $\psi_{\mathrm{wb}} \mathrm{M}=-0,61 \mathrm{MPa}$ e $\sigma=0,348$ MPa. Rowse \& Finch-Savage (2003), em testes realizados com sementes de Daucus carota L. e Allium cepa L., observaram que o $\psi_{\mathrm{wb}} \mathrm{M}$ pode aumentar linearmente com a temperatura, dependendo da faixa 
térmica, o que pode ser o caso de sementes de $D$. cordata, embora testes com maior número de temperaturas se façam necessários para se confirmar isso. Usando-se o $\psi_{\mathrm{wb}}$ como parâmetro, foram constatadas que as sementes de $D$. cordata são menos sensíveis ao $\psi_{\mathrm{w}}$ à temperatura de $19{ }^{\circ} \mathrm{C}$ do que a $25^{\circ} \mathrm{C}$, já que os valores de $\psi_{\text {wb }}$ foram mais negativos à $19^{\circ} \mathrm{C}$. A $32^{\circ} \mathrm{C}$, o $\psi_{\text {wb }}$ ocupou uma posição mais ou menos intermediária em relação aos dois outros tratamentos térmicos. Larsen et al. (2004) observaram que o $\psi_{\mathrm{wb}}$ aumentou com a temperatura em Poa pratensis L., e decresceu em Lolium perenne L. Esses autores reportam que essa interação entre potencial hídrico e temperatura pode afetar a eficácia de modelos que trabalham esses dois fatores em conjunto, como o de $\psi_{\mathrm{w}}$. grau dia (hydrothermal time) (Gummerson, 1986), com o objetivo de se prever o comportamento germinativo das sementes no campo.

O parâmetro $\theta_{\mathrm{H}}$ representa a quantidade de $\psi_{\mathrm{w}}$ dia necessária para cada semente completar a germinação, sendo que, quanto maior $\theta_{\mathrm{H}}$, menor a velocidade do processo por unidade de $\psi_{\mathrm{w}}$ acima de seu $\psi_{\mathrm{wb}}$. Em outras palavras, para um dado $\psi_{\mathrm{w}}$, o tempo que uma semente leva para germinar é inversamente proporcional à diferença entre o $\psi_{\mathrm{w}}$ e o seu $\psi_{\mathrm{wb}}$ (Bradford 1995). Em D. cordata, o valor de $\theta_{\mathrm{H}}$ foi maior para sementes germinadas à temperatura sub-ótima, e menor a $25^{\circ} \mathrm{C}$, mostrando que em temperatura ótima a velocidade de germinação num determinado $\psi_{\mathrm{w}}$ é maior. Em temperatura supra-ótima, a quantidade de $\psi_{\mathrm{w}}$ dia necessária para a germinação foi maior do que a $25^{\circ} \mathrm{C}$, e menor do que a $19{ }^{\circ} \mathrm{C}$. Portanto, em temperaturas baixas, para um dado $\psi_{\mathrm{w}}$, um maior tempo é necessário para que a semente acumule uma quantidade de $\theta_{\mathrm{H}}$ necessária para a germinação de uma dada porcentagem. Com exceção da fração $5 \%$, não foram constatadas diferenças significativas entre as diferentes subpopulações (representadas pelas diferentes frações percentuais) com relação a $\theta_{\mathrm{H}}$, enquanto Wang et al. (2005) relatam que, em sementes de Eurotia lanata (Pursh) Moq., $\theta_{\mathrm{H}}$ é constante entre diferentes subpopulações apenas em temperaturas ótimas, ocorrendo um aumento linear de $\theta_{\mathrm{H}}$ com o aumento da fração percentual em temperaturas sub-ótimas. Assim, como relatado para algumas espécies não cultivadas (Huarte \& Benech-Arnold 2005), o modelo descreveu com razoável precisão a distribuição das porcentagens acumuladas de germinação de $D$. cordata, especialmente nas temperaturas sub-ótima e supra-ótima. A $25^{\circ} \mathrm{C}$, talvez pelo fato da germinação não ter se estabilizado nos tratamentos com diferentes $\psi_{\mathrm{w}}$, mesmo após 30 dias de experimento, a eficácia do modelo foi relativamente menor. Esse aspecto também fica evidenciado quando as curvas de germinação nos diferentes potenciais hídricos são normalizadas para $\psi_{\mathrm{w}}=0$, ou seja, para a curva que seria esperada se as sementes estivessem em água destilada, conforme proposto por Bradford (1995). Considerando-se que essa normalização também é uma indicação da eficiência do modelo em descrever o comportamento germinativo em resposta a diferentes potenciais hídricos (Dahal \& Bradford 1990), isso corrobora a validade da utilização do modelo para descrever a resposta de sementes de Drymaria cordata a variações do $\psi_{\mathrm{w}}$ do meio, particularmente em temperaturas sub- e supra-ótimas, onde a coincidência dos pontos em relação às curvas esperadas foi maior.

Agradecimentos - Victor J. M. Cardoso agradece ao Conselho Nacional de Desenvolvimento Científico e Tecnológico pela bolsa de Produtividade em Pesquisa durante a elaboração deste trabalho.

\section{Referências bibliográficas}

BATLLA, D. \& BENECH-ARNOLD, R.L. 2006. The role of fluctuations in soil water content on the regulation of dormancy changes in buried seeds of Polygonum aviculare. Seed Science and Research 16:47-59.

BEWLEY, J.D. 1997. Seed germination and dormancy. Plant Cell 9:1055-1066.

BRADFORD, K. 1995. Water relations in seed germination. In Seed development and germination J. Kigel \& G. Galili, eds.). Marcel Dekker Inc, New York, p.351396.

BRADFORD, K. 2002. Applications of hydrothermal time to quantifying and modeling seed germination and dormancy. Weed Science 50:248-260.

DAHAL, P. \& BRADFORD, K. 1990. Effects of priming and endosperm integrity on seed germination rates of tomato genotypes. II. Germination at reduced water potential. Journal of Experimental Botany 41:14411453.

DAHAL, P. \& BRADFORD, K. 1994. Hydrothermal time analysis of tomato seed germination at subopitmal temperature and reduced water potential. Seed Science and Research 4:71-80.

DUMUR, D., PILBEAM, C.J. \& CRAIGON, J. 1990. Use of the Weibull function to calculate cardinal temperatures in faba bean. Journal of Experimental Botany 41:14231430.

EL SIDDIG, K., INANAGA, S., ALI, A.M., AN, P., GEBAUER, J. \& EBERT, G. 2004. Response of Tamarindus indica $\mathrm{L}$. to iso-osmotic solutions of $\mathrm{NaCl}$ and PEG during germination. Journal of Applied Botany 78:1-4. 
FINCH-SAVAGE, W.E. \& PHELPS, K. 1993. Onion (Allium сера L.) seedling emergence patterns can be explained by the influence of soil temperature and water potential on seed germination. Journal of Experimental Botany 44:407-414.

GUMMERSON, R.J. 1986. The effect of constant temperatures and osmotic potentials on the germination of sugar beet. Journal of Experimental Botany 37:729-741.

HUARTE, R. \& BENECH-ARNOLD, R. 2005. Incubation under fluctuating temperatures reduces mean base water potential for seed germination in several non-cultivated species. Seed Science and Research 15:89-97.

LABOURIAU, L.G. 1983. A germinação das sementes. Secretaria Geral da Organização dos Estados Americanos, Washington D.C.

LARSEN, S.U., BAILLY, C., COME, D. \& CORBINEAU, F. 2004. Use of the hydrothermal time model to analyse interacting effects of water and temperature on germination of three grass species. Seed Science and Research 14:35-50.

LORENZI, H. 1982. Plantas daninhas do Brasil: terretres, aquáticas, parasitas e tóxicas. Edição do Autor, Nova Odessa.

MICHEL, B.E \& KAUFMANN, M.R. 1973. The osmotic potential of polyethylene glycol 6000. Plant Physiology 51:914-916.
ROWSE, H.R. \& FINCH-SAVAGE, W.E. 2003. Hydrothermal threshold models can describe the germination response of carrot (Daucus carota) and onion (Allium сера) seed populations across both sub- and supraoptimal temperatures. The New Phytologist 158: 101-108.

SPRINGER, T.L. 2005. Germination and early seedling growth of chaffy-seeded grasses at negative water potentials. Crop Science 45:2075-2080.

TILKI, F. 2005. Seed germination and radicle development in six provenances of Pinus sylvestris L. under water stress. Israel Journal of Plant Sciences 53:29-33.

TOSELLI, M.E. \& CASANAVE, E.C. 2005. Hydropriming and cottonseed germination under unfavourable conditions: modifications in hydrotime model parameters. Seed Science and Technology 33:8796.

WANG, R., BAI, Y. \& TANINO, K. 2005. Germination of winterfat (Eurotia lanata) seeds at reduced water potentials: testing assumptions of hydrothermal time model. Environmental and Experimental Botany 53: 49-63.

WELBAUM, G.E., BRADFORD, K., YIM, K., BOOTH, D.T. \& OLUOCH, M.O. 1998. Biophysical, physiological and biochemical processes regulating seed germination. Seed Science and Research 8:161-172. 